\title{
Anterior cruciate ligament reconstruction with bone-patellar tendon-bone grafts: a prospective comparative study of femoral cortical suspensory fixation and interference screw fixation
}

\author{
Sang-Woo Jeon ${ }^{1,2}$, Min Jung ${ }^{1,2}$, Yong-Min Chun ${ }^{1,2}$, Sangyun $\mathrm{Oh}^{1}$, Chong Hyuk Choi ${ }^{1,2}$, Sung-Jae Kim ${ }^{3}$, \\ Sung-Hwan $\mathrm{Kim}^{1,2}$ \\ ${ }^{I}$ Department of Orthopedic Surgery, Yonsei University College of Medicine, ${ }^{2}$ Arthroscopy \& Joint Research Institute, Yonsei University \\ Health System, ${ }^{3}$ Department of Orthopedic Surgery, Gangnam Yonsei Sarang Hospital, Seoul, Korea
}

\begin{abstract}
Background: We prospectively compared the clinical outcome between suspensory fixation and conventional interference screw fixation of bone-patellar tendon-bone (BPTB) grafts in patients who underwent anterior cruciate ligament (ACL) reconstruction.

Methods: We enrolled 32 patients who underwent primary ACL reconstruction with BPTB grafts. The minimum followup period was 24 months. We categorized subjects into two groups based on treatment: 14 patients into the interference screw fixation group and 12 patients into the suspensory fixation group. The patients were also analyzed in terms of patellar tendon length. We assessed clinical outcome with the Lachman test and the pivot shift test and anterior translation with a KT-2000 arthrometer. We assessed functional outcome in terms of the Lysholm score, the International Knee Documentation Committee subjective score, and objective grades. In the suspensory group, we measured the length from the outer cortical aperture to the femoral grafted bone and the degree of axial rotation of the grafted bone by using computed tomography (CT) scans taken immediately and 3 months postoperatively.

Results: We did not observe a statistically significant difference in anterior translation between the two groups $(\mathrm{P}=0.934)$. We found that the patients who underwent suspensory fixation had an average difference in length between the graft bone and the cortex bone of $0.06 \mathrm{~mm}$ (range, -0.34 to $0.46 \mathrm{~mm} ; \mathrm{P}=0.755$ ) and axial rotation of $0.57^{\circ}$ (range, $-0.76^{\circ}$ to $1.89^{\circ}$; $\mathrm{P}=0.366)$.

Conclusion: In sum, we found that the degree of postoperative anterior translation in patients who received femoral suspensory fixation was comparable to those who underwent interference screw fixation. In addition, we did not observe early loosening of the graft-bone block upon CT analysis. Therefore, we conclude that suspensory fixation could be an alternative to the conventional interference screw fixation technique that can eliminate the graft-tunnel mismatch observed in BPTB grafting in ACL reconstruction.
\end{abstract}

Keywords: Anterior cruciate ligament reconstruction; Bone-patellar tendon-bone; Length mismatch; Suspensory

\section{INTRODUCTION}

The choice of graft for anterior cruciate ligament (ACL) reconstruction remains controversial and depends largely on the preference of surgeons. The historically accepted gold standard of choice is the autologous bone-patellar tendon-bone (BPTB) graft. Recent biomechanical studies [1-3] have reported that the biomechanics of the ACL can be effectively restored when the femoral tunnel is created in the 10 or 2 o'clock position as opposed to the conventional 11 or 1 o'clock position during ACL reconstruction. However, the horizontal fixation of the femoral tunnel
Received October 30, 2017; Revised November 23, 2017; Accepted November 30, 2017

$\triangle$ Correspondence to: Sung-Hwan Kim, Department of Orthopedic Surgery, Gangnam Severance Hospital, 211 Eonju-ro, Gangnamgu, Seoul 06273, Korea. Tel: +82-2-2019-3415, Fax: +82-2-573-5393, E-mail: orthohwan@gmail.com

Copyright (C) 2018 Korean Arthroscopy Society and Korean Orthopedic Society for Sports Medicine. All rights reserved.

(a) This is an open-access article distributed under the terms of the Creative Commons Attribution Non-Commercial License (http://creativecommons.org/licenses/ by-nc/4.0) which permits unrestricted noncommercial use, distribution, and reproduction in any medium, provided the original work is properly cited. 
is likely to cause a greater graft-tunnel mismatch than the conventional vertical fixation. Moreover, the use of interference screws at the intra-articular aperture of the femoral tunnel during graft fixation has been shown to exacerbate the graft-tunnel mismatch $[4,5]$.

Femoral cortical suspension devices such as EndoButtons (Smith \& Nephew Endoscopy, Andover, MA, USA) can be used to adjust and match the length of the BPTB graft to the anatomical femoral tunnel [6]. Tecklenburg et al. [7] reported satisfactory outcomes after a 2- to 5-year follow-up in patients who received Endobutton femoral cortical suspensory fixation with autogenous patellar tendon-bone grafts. However, no prospective studies have compared the outcomes between femoral cortical suspensory fixation and conventional interference screw fixation for anatomical ACL reconstruction. The purpose of this prospective study was to compare the outcomes between these two fixation techniques. We also evaluated the frequency and the distance of early graft loosening after suspensory fixation through a multi-planar computed tomography (CT) analysis at two time-points: immediately and 3 months postoperatively. We hypothesized that there would be no significant differences in postoperative knee stability and functional outcomes between the two treatment groups and that the femoral cortical suspensory fixation technique would not lead to early loosening of the graft-bone block.

\section{METHODS}

\section{Patients}

The study was performed after approval from the institutional review board of the Gangnam Severance Hospital, Yonsei University College of Medicine (IRB no. 4-20100781), and signed consent forms were obtained from the patients before the study began. From 2010 through to 2012, we prospectively assessed the eligibility of patients who were scheduled to have ACL reconstruction in the Gangnam Severance Hospital, Yonsei University College of Medicine (Seoul, Korea). The inclusion criteria for the study were as follows: patients aged over 20 years, a clinical and arthroscopic diagnosis of a primary ACL rupture, and ACL reconstruction using an autologous BPTB graft. We excluded 144 patients from the 176 patients who were eligible for the following reasons: a partial ACL tear in 3 patients; a non-BPTB graft in 35 patients (a hamstring graft in 34 and an allogenous graft in 1); revisional reconstruction in 11 patients; an accompanying ligament injury on either knee in 18 patients; an indication for sub-total or total meniscectomy on the affected knee in 7 patients; an accompanying articular cartilage defect of Outerbridge grade II or above in 6 patients; and refusal to sign consent forms in 32 patients. Using a threshold of 45 $\mathrm{mm}$, we classified the patients into two groups in terms of patellar tendon length, which we measured intraoperatively. We used a 20-mm bone block from the BРTB graft because the intra-articular length of the ACL has been shown to be between 25 and $30 \mathrm{~mm}$ [8] and the tibial tunnel length, between 30 and $40 \mathrm{~mm}$. Our experiences suggest that graft-tunnel mismatches in length are more likely if the total length of the patella tendon and the bone block exceeds $65 \mathrm{~mm}$. Among the 48 patients with a patellar tendon length less than $45 \mathrm{~mm}, 16$ patients were allocated consecutively and 32 patients were excluded. Because the required sample size was 16 per group. We allocated 16 patients each into the interference screw fixation group and the suspensory fixation group. Over a follow-up period of at least 24 months, the postoperative outcomes were evaluated in 14 patients from the interference screw fixation group and in 12 patients from the suspensory fixation group (a total of 6 patients were lost to follow-up) (Fig. 1).

\section{Surgical technique}

All operations were conducted by a single surgeon at a single facility. To harvest the patella, we first made a longitudinal skin incision approximately $7 \mathrm{~cm}$ long from the inferiomedial margin of the patella to the insertion site of the pes anserinus tendon. We harvested the middle third of the patellar tendon, which includes the patella and the tibial tubercle bone. We made them into rectangular bone blocks measuring $20 \mathrm{~mm}$ by $10 \mathrm{~mm}$. We placed the tibial tubercle bone block within the 9-mm-diameter femoral tunnel and the patellar bone block within the 10-mm-diameter tibial tunnel. We connected the bone flap within the femoral tunnel to an EndoButton to drill a 2.5-mm-diameter hole, which is $7 \mathrm{~mm}$ from the end, and placed two differently colored No. 2 Fiberwire (Arthrex, Naples, FL, USA) loops through this hole. These sutures were configured in a way that they could be tied when pulled towards the Endobutton (Fig. 2). All graft tendons were pre-conditioned under $80 \mathrm{~N}$ of tension, exerted through the GraftMaster (Smith \& Nephew Endoscopy), for 20 minutes before grafting. We used a high anterolateral portal as the viewing portal (through which we used a $70^{\circ}$ arthroscope) and a low anteromedial portal as the 


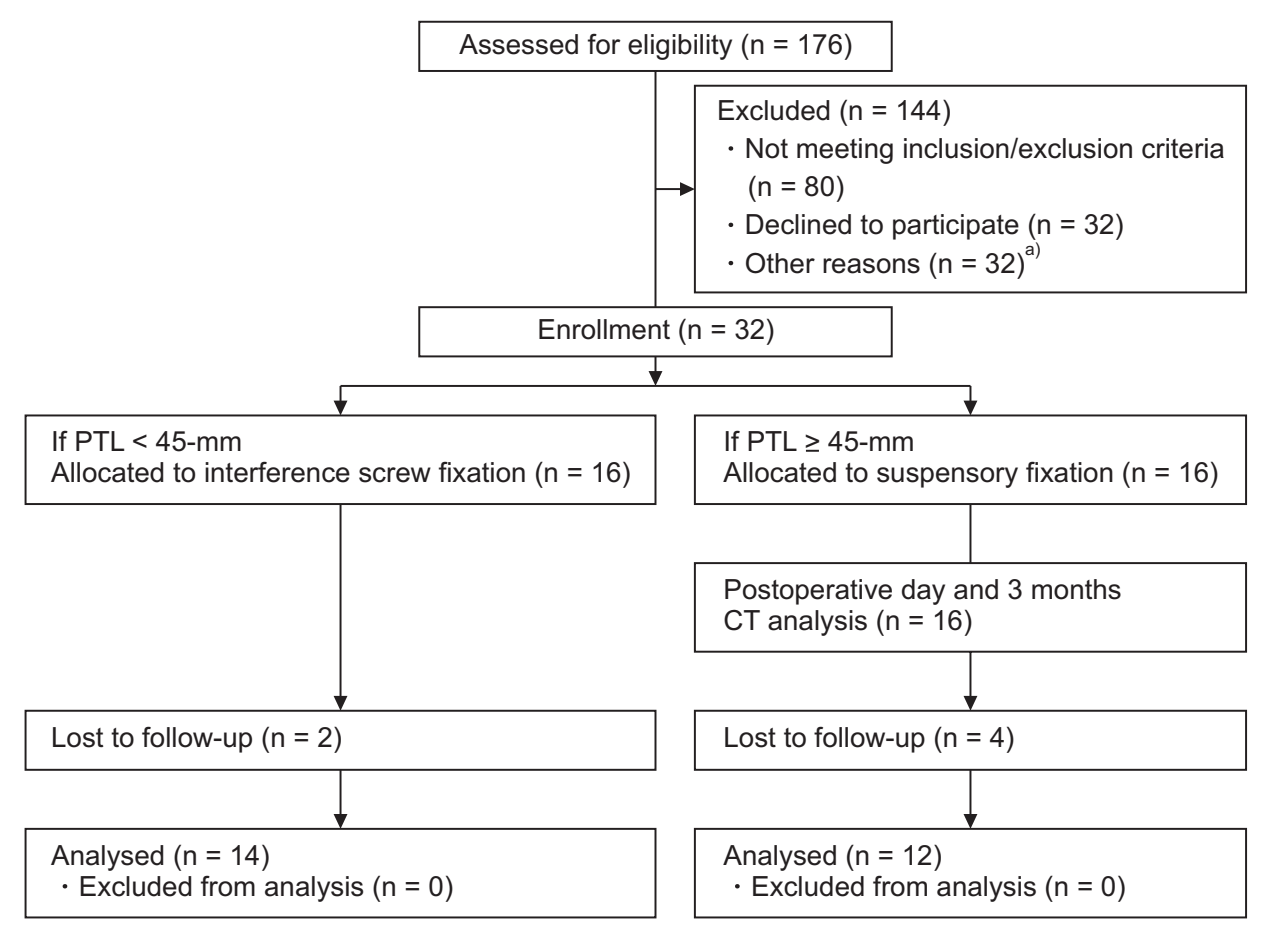

Fig. 1. Enrollment, assignment, and follow-up diagram. PTL, patella tendon length. ${ }^{\text {a) }}$ Among the 48 patients with PTL less than $45 \mathrm{~mm}, 16$ patients required sample size per group were allocated consecutively and 32 patients were excluded.

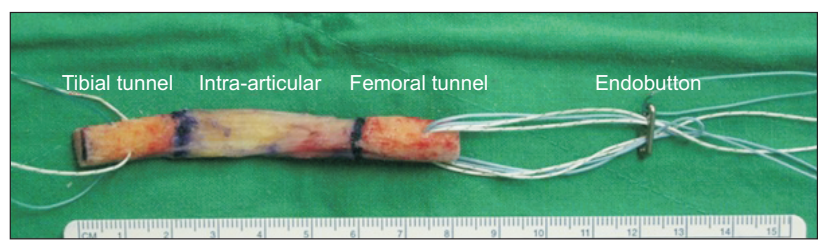

Fig. 2. Photograph of a prepared bone-patellar tendon-bone autograft.

working portal [9]. We performed anatomical reconstruction with the modified transtibial method [10]. A tibial guide (Acufex; Smith \& Nephew Endoscopy) tilted to $55^{\circ}$ was inserted through the anteromedial portal. We reamed a 10-mm-diameter tibial tunnel along the guide pin that passed from the anterior border of the medial collateral ligament and 4 to $5 \mathrm{~cm}$ below the anteromedial joint line to the center of the ACL footprint. The 7-mm femoral offset guide (Arthrex) was externally rotated within the tibial tunnel, and the femoral guide pin was pierced into the center of the ACL footprint. We drilled through the femoral cortical bone with an EndoButton reamer. Using a depth gauge, we measured the distance between the tibial tunnel entrance and the lateral femoral cortex exit $(\mathrm{H})$ and the distance between the femoral tunnel aperture and the lateral femoral cortex (f). After measuring the total length of the BPTB graft (p), we calculated the length of the femoral tunnel to be reamed as $(\mathrm{D})=\mathrm{f}-(\mathrm{H}-\mathrm{p})$ (Fig. 3). We reamed the femoral tunnel, alternating between a

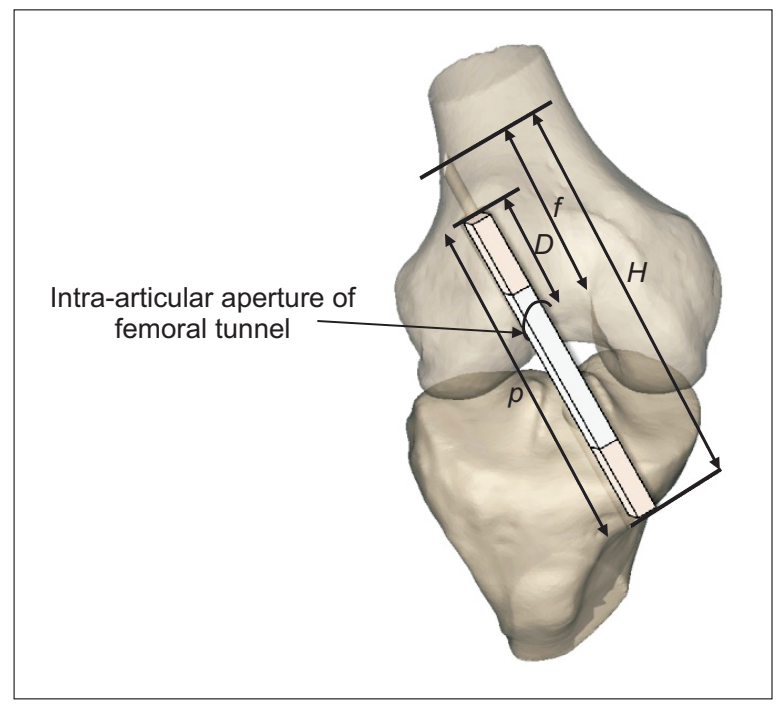

Fig. 3. Diagram explains how the length of femoral tunnel was calculated. The length of the femoral tunnel is $(D)=f-(H-p)$. H, the distance between the tibial tunnel entrance and the lateral femoral cortex exit; $f$, the distance between the femoral tunnel aperture and the lateral femoral cortex; $p$, after measuring the total length of the bone-patellar tendon-bone graft.

6-mm and a 9-mm headed reamer, along the reinserted guide pin. We passed the head of the reamer with the patient's knee under slight extension to prevent the reamer head from damaging the intra-articular posterior margin of the tibial tunnel. We then engaged the reamer with the 
knee at $90^{\circ}$ of flexion [11]. We made a $1-\mathrm{cm}$ stab wound next to the guide pin on the lateral proximal thigh and inserted the plastic sheath from the outside so that it made contact with the lateral femoral cortex. We threaded the two No. 2 Fiberwires of the Endobutton through the eye of the guide pin and knotted them at the outside of the tibial tunnel. We pulled the guide pin from the tibial tunnel towards the femoral tunnel until it emerged into the femoral cortex, placing tension on the Endobutton. This was confirmed using a $30^{\circ}$ arthroscope sealed with a plastic sheath. We tensioned the two Fiberwires and knotted them with a knot pusher (Fig. 4). We connected the No. 2 Ethibonds (Ethicon, Somerville, NJ, USA), passing through the bone block of the tibial side of the graft, to the $\mathrm{SE}^{\mathrm{TM}}$ Graft Tensioner (Linvatec, Largo, FL, USA) placed at the tibial tunnel entrance. For the tibial tunnel fixation, we performed 20 cycles of pre-tensioning and then inserted a bio-absorbable interference screw (Genesys ${ }^{\circledR}$; Linvatec) into the cancellous bone under 80 $\mathrm{N}$ of tension and with the knee at $15^{\circ}$ of flexion. For the
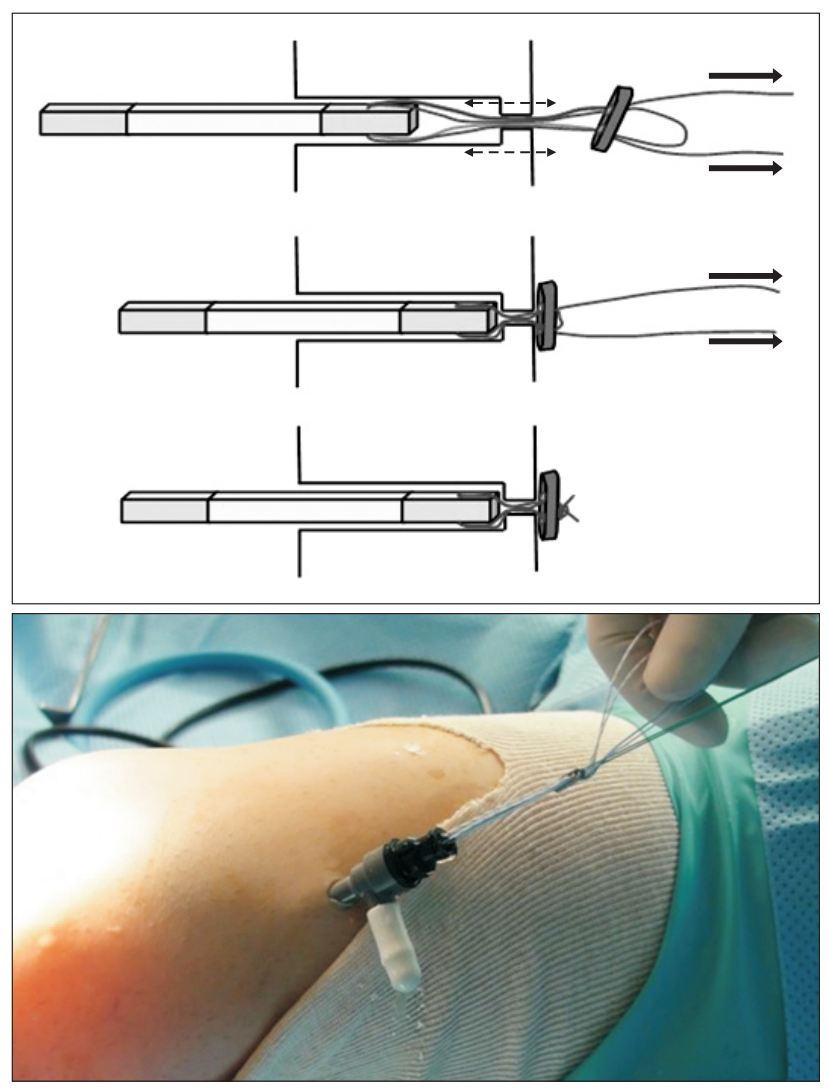

Fig. 4. Free space in the femoral tunnel was reduced by dragging fiberwires. Two fiberwires were strained and procedure was finished by making knots. interference screw fixation group, the femoral tunnel was reamed to a depth of $25 \mathrm{~mm}$, and the bone block was secured in the femoral socket by inserting a $7 \times 20$-mm bioabsorbable interference screw (Genesys ${ }^{\circledR}$ ) with the knee at $110^{\circ}$ of knee flexion. All other procedures between the two groups were identical.

\section{Postoperative rehabilitation}

We allowed patients to perform partial weight bearing with a hinged ACL brace for 6 postoperative weeks and total weight-bearing within the capacity of each patient thereafter. The patients were able to fully extend their leg immediately postoperatively and flex at $120^{\circ}$ after 4 weeks of range of motion (ROM) exercises. Closed chain exercises were permitted immediately postoperatively and jogging, from the 4 th postoperative month. From the 7th postoperative month, the patients returned to full exercise only when the quadriceps power of the affected leg was more than $80 \%$ of that of the unaffected contralateral leg.

\section{Clinical assessments}

Preoperatively, we collected data on demographic variables, such as patient age, sex, height, body mass index, affected side, period from injury to the operation, and generalized laxity score according to the Beighton-Horan index [12] (Table 1). Patellar tendon length was measured intraoperatively. We evaluated the clinical outcome of the affected knee relative to the contralateral unaffected knee by using the Lachman test (to manually measure ACL laxity), the pivot-shift test, and ROMs of the knee at two time-points: immediately postoperatively and 24 months postoperatively. We compared the KT-2000 arthrometer (MEDmetric, San Diego, CA, USA) measurements of the affected knee and those of the normal contralateral knee to calculate the side-to-side difference (SSD) under manual maximal force. To test extensor function of the quadriceps, we conducted the one-leg hop test and the Biodex machine (Biodex Medical System, Shirley, NY, USA) preoperatively and at the 24-month follow-up. The one-leg hop was calculated as the average of two measurements for each limb. The limb symmetry index was calculated as the percentage of the averaged hop distance of the affected limb divided by the averaged hop distance of the unaffected limb. The Biodex machine was performed at velocities $60^{\circ}$ and $180^{\circ}$ per second five times each. Peak torque deficit was the primary variable of our study. Clinical outcome was measured using the follow- 
Table 1. Demographic data for patients

\begin{tabular}{lccc}
\hline \multicolumn{1}{c}{ Variable } & Group IF (n=14) & Group SF (n=12) & P-value \\
\hline Age (yr) & $29.1 \pm 9.8$ & $33.1 \pm 14.9$ & 0.441 \\
Sex (male/female) & $14 / 0(100 / 0)$ & $11 / 1(91.7 / 8.3)$ & 0.462 \\
Affected side (right/left) & $6 / 8(42.9 / 57.1)$ & $5 / 7(41.7 / 58.3)$ & 0.951 \\
Duration from injury to operation (wk) & $11.1 \pm 7.4$ & $12.4 \pm 10.9$ & 0.742 \\
Concomitant operation & & & 0.849 \\
None & $10(71.4)$ & $7(58.3)$ & $2(16.7)$ \\
Partial meniscectomy & $1(7.1)$ & $3(25.0)$ & 0.342 \\
Meniscal repair & $3(21.4)$ & $175.1 \pm 9.7$ & 0.992 \\
Height (cm) & $171.8 \pm 5.5$ & $25.1 \pm 3.0$ & 0.678 \\
BMI (kg/m ${ }^{2}$ ) & $25.1 \pm 3.3$ & $2.5(0-5)$ & $<0.0001$ \\
Generalized laxity (0-9) & $2(0-9)$ & $48.0 \pm 3.7$ & \\
Patellar tendon length $(\mathrm{mm})$ & $39.8 \pm 1.9$ & & \\
\hline
\end{tabular}

Values are presented as mean \pm standard deviation, number only, number (\%), or mean (range).

The sum of the percentages does not equal $100 \%$ because of rounding.

IF, interference screw fixation; SF, suspensory fixation; BMI, body mass index.

ing assessment tools: the 100-mm visual analogue scale (VAS), the Lysholm score, the Tegner activity, and the International Knee Documentation Committee (IKDC) subjective/objective knee evaluation. Questionnaires for the IKDC evaluation were filled out by the patients. Physical examination was independently performed by two trained orthopedic surgeons.

\section{Computed tomography assessment}

We performed CT of the suspensory fixation group on the operation day and at 3 months after the operation. CT scans were sliced at $0.625-\mathrm{mm}$ intervals, and a 16-channel CT scanner (GE Healthcare, Buckinghamshire, UK) was used with the patient's knee at full extension. We imported the Digital Imaging and Communications in Medicine data from a picture archiving and communication system into the OsiriX imaging software version 5.8.2 (Pixmeo, Geneva, Switzerland). We measured early graft stability in terms of the distance between the cortex and the bone block (CBD) and the rotational angle of the bone block (RAB) on two-dimensional multi-plane CT scans. We measured CBD as the distance from the femoral lateral cortex to the proximal end of the femoral bone block on coronal plane, which includes the central axis (line $\mathrm{C}$ ) of the femoral tunnel (Fig. 5). We measured RAB as the angle formed from the intersection between the epicondylar axis (line A) and the horizontal axis (line B) of the femoral bone block on axial plane, which contains a cross-section of the femoral tunnel (Fig. 5). The angle was measured in the clockwise or counterclockwise direction
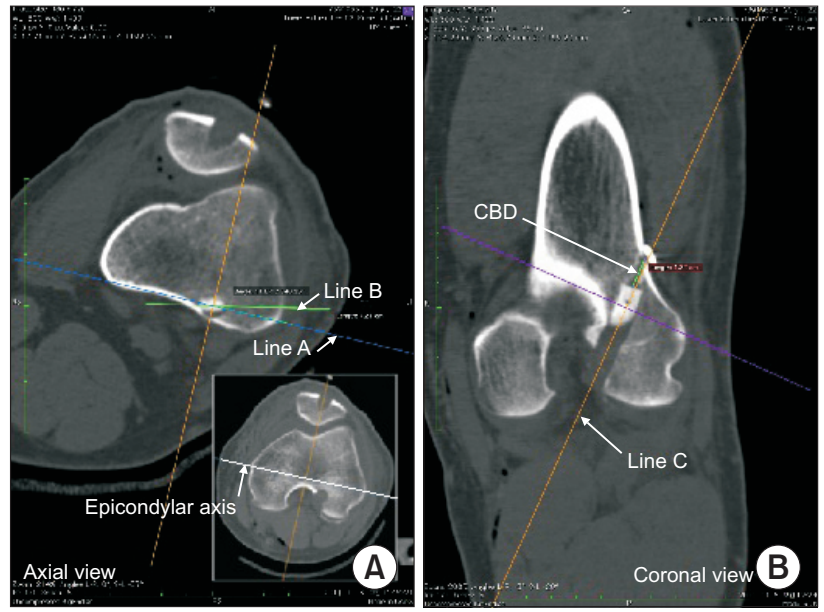

Fig. 5. (A) RAB: angle formed from the epicondylar axis to the length axis of the bone block in the femoral tunnel on the (axial view). (B) CBD: the distance from the lateral cortex to the proximal part of the bone block inside the femoral tunnel on the (coronal view).

when the lesion was on the left or right knee, respectively.

\section{Statistical methods}

We calculated the sample size by using parameters such as the manual maximal SSD derived from the KT-2000 arthrometer. The criterion for non-inferiority of the suspensory group was an upper limit of the one-sided 95\% confidence interval (CI) for the difference in anterior translation of less than $1.5 \mathrm{~mm}$ (a 1.5-mm standard deviation based on previous studies) with a significance (alpha) level of 5\% and a 1-beta (power) of $80 \%$ [13-15]. Our analysis revealed a sample size of 13 patients for each 
group, therefore, requiring an initial registration of 16 patients in case of the approximately $20 \%$ of patients who may be lost to follow-up [16]. We used either the twosample t-test or the Mann-Whitney U-test for continuous and ordinal variables and the chi-square or the Fisher's exact test for categorical variables in a normally distributed sample. We used paired t-tests to analyze CBD and $\mathrm{RAB}$ of the suspensory fixation group. We summarized data as either the mean and standard deviation or median and range. Statistical analyses were performed using the IBM SPSS Statistics ver. 22.0 (IBM Co., Armonk, NY, USA), and a P-value of $<0.05$ was considered statistically significant.

\section{RESULTS}

We found that the mean duration from injury to treatment was $11.1 \pm 7.4$ weeks in the interference screw fixation group and $12.4 \pm 10.9$ weeks in the suspensory fixation group. We found that the mean length of the patellar tendon was significantly shorter in the interference screw fixation group than the suspensory fixation group (39.8 \pm 1.9 mm vs. $48.0 \pm 3.7 \mathrm{~mm}$, respectively; $\mathrm{P}<0.0001)$. The demographic variables did not show a statistically significant difference between the two groups (Table 1). The average age of the patients was $29.1 \pm 9.8$ and $33.1 \pm$ 14.9 years, respectively. Only one patient in the suspensory fixation group was female.

\section{Clinical assessment}

We found that the mean preoperative SSD in anterior translation in the interference screw fixation group and the suspensory fixation group was $5.9 \pm 1.5 \mathrm{~mm}$ and 5.9 $\pm 2.9 \mathrm{~mm}$, respectively. Neither the preoperative SSD nor other parameters of outcome reveal a statistically significant difference between the two groups (Table 2). The degree of anterior translation was $1.1 \pm 2.2 \mathrm{~mm}$ and $1.0 \pm$ $1.5 \mathrm{~mm}$, respectively. Although the SSD at the 24-month follow-up decreased in both groups, the differences were insignificant $(\mathrm{P}=0.934)$. The mean difference in the 24-month follow-up SSD between the two groups was $-0.52 \mathrm{~mm}$ and the $95 \% \mathrm{CI}$ ranged between $-1.59 \mathrm{~mm}$ and

Table 2. Comparison of preoperative variables

\begin{tabular}{|c|c|c|c|}
\hline Variable & Group IF $(n=14)$ & Group SF $(n=12)$ & P-value \\
\hline SSD of KT-2000 (mm) & $5.9 \pm 1.5$ & $5.9 \pm 2.9$ & NS \\
\hline Lachman test grade & & & NS \\
\hline $3-5 \mathrm{~mm}$ & $4(28.6)$ & $4(33.3)$ & \\
\hline $6-10 \mathrm{~mm}$ & $10(71.4)$ & $7(58.3)$ & \\
\hline$>10 \mathrm{~mm}$ & $0(0)$ & $1(8.3)$ & \\
\hline Range of motion deficit $\left({ }^{\circ}\right)$ & $19.1 \pm 28.4$ & $26.3 \pm 26.1$ & NS \\
\hline Pivot shift test grade & & & NS \\
\hline Normal & $2(14.3)$ & $1(8.3)$ & \\
\hline Grade 1 & $8(57.1)$ & $4(33.3)$ & \\
\hline Grade 2 & $4(28.6)$ & $6(50.0)$ & \\
\hline Grade 3 & $0(0)$ & $1(8.3)$ & \\
\hline One-leg hop test (\%) & $29.1 \pm 26.5$ & $19.7 \pm 24.2$ & NS \\
\hline Visual analogue scale $(0-100)$ & $29.1 \pm 25.7$ & $44.6 \pm 29.2$ & NS \\
\hline Lysholm score & $53.4 \pm 15.7$ & $42.1 \pm 27.7$ & NS \\
\hline Tegner activity scale & $6.2 \pm 1.6$ & $5.7 \pm 1.6$ & NS \\
\hline IKDC subjective score & $43.4 \pm 16.3$ & $35.4 \pm 17.6$ & NS \\
\hline IKDC objective grade & & & NS \\
\hline B & $3(21.4)$ & $2(16.7)$ & \\
\hline $\mathrm{C}$ & $10(71.4)$ & $6(50.0)$ & \\
\hline $\mathrm{D}$ & $1(7.1)$ & $4(33.3)$ & \\
\hline
\end{tabular}

Values are presented as mean \pm standard deviation or number (\%).

The sum of the percentages does not equal $100 \%$ because of rounding.

IF, interference screw fixation; SF, suspensory fixation; SSD, side-to-side difference; IKDC, International Knee Documentation Committee; NS, nonsignificant. 
Table 3. Comparison of postoperative variables

\begin{tabular}{|c|c|c|c|}
\hline Variable & Group IF $(n=14)$ & Group SF (n = 12) & P-value \\
\hline SSD of KT-2000 (mm) & $1.1 \pm 2.2$ & $1.0 \pm 1.5$ & NS \\
\hline Lachman test grade & & & NS \\
\hline$<3 \mathrm{~mm}$ & $12(85.7)$ & $9(75.0)$ & \\
\hline $3-5 \mathrm{~mm}$ & $2(14.3)$ & $3(25.0)$ & \\
\hline Range of motion deficit $\left(^{\circ}\right)$ & $1.9 \pm 5.0$ & $1.4 \pm 2.3$ & NS \\
\hline Pivot shift test grade & & & NS \\
\hline Normal & $13(92.9)$ & 10 (83.3) & \\
\hline Grade 1 & $1(7.1)$ & $2(16.7)$ & \\
\hline One-leg hop test (\%) & $81.9 \pm 14.9$ & $83.9 \pm 15.1$ & NS \\
\hline Visual analogue scale (0-100) & $8.8 \pm 9.4$ & $16.3 \pm 10.6$ & NS \\
\hline Lysholm score & $89.4 \pm 15.2$ & $79.2 \pm 12.0$ & NS \\
\hline Tegner activity scale & $4.4 \pm 1.6$ & $3.6 \pm 1.5$ & NS \\
\hline IKDC subjective score & $75.2 \pm 13.0$ & $66.8 \pm 13.7$ & NS \\
\hline IKDC objective grade & & & NS \\
\hline A & $10(71.4)$ & $8(66.7)$ & \\
\hline B & $4(28.6)$ & $4(33.3)$ & \\
\hline \multicolumn{4}{|c|}{ Peak torque deficit (quadriceps, \%) } \\
\hline $60^{\circ} / \mathrm{s}$ & $23.7 \pm 23.3$ & $31.7 \pm 18.9$ & NS \\
\hline $180^{\circ} / \mathrm{s}$ & $24.1 \pm 25.7$ & $34.9 \pm 15.0$ & NS \\
\hline
\end{tabular}

Values are presented as mean \pm standard deviation or number (\%).

IF, interference screw fixation; SF, suspensory fixation; SSD, side-to-side difference; IKDC, International Knee Documentation Committee; NS, nonsignificant.

Table 4. Measurements of computed tomography parameters in the suspensory fixation group

\begin{tabular}{cccccc}
\hline Variable & Immediately after operation & 3 mo after operation & Mean differences & 95\% CI & P-value \\
\hline $\mathrm{CBD}(\mathrm{mm})$ & $12.0 \pm 4.7$ & $11.9 \pm 4.8$ & 0.06 & -0.34 to 0.46 & NS \\
$\mathrm{RAB}\left({ }^{\circ}\right)$ & $26.6 \pm 14.6$ & $26.0 \pm 14.6$ & 0.57 & -0.76 to 1.89 & NS \\
\hline
\end{tabular}

Values are presented as mean \pm standard deviation.

$\mathrm{CI}$, confidence interval; CBD, the distance between the cortex to the bone block; RAB, measured the rotational angle of the bone block; NS, non-significant.

$1.48 \mathrm{~mm}$, showing that the suspensory fixation group met the non-inferiority criterion with respect to anterior laxity. The outcomes of ROM exercises, the pivot-shift test, VAS, the Lysholm score, the Tegner score, and the IKDC subjective and objective rating did not significantly differ between the two groups (Table 3). The peak torque deficit of the quadriceps measured using the Biodex tests at the 24-month follow-up did not significantly differ between the two groups in either velocities: $60^{\circ} / \mathrm{s}(23.7 \% \pm 23.3 \%$ vs. $31.7 \% \pm 18.9 \%$, respectively) or $180^{\circ} / \mathrm{s}(24.1 \% \pm 25.7 \%$ vs. $34.9 \% \pm 15.0 \%$, respectively) (Table 3 ).

\section{Computed tomography findings}

We found that the difference in the mean $\mathrm{CBD}$ and $\mathrm{RAB}$ in the suspensory fixation group between the immediate and 3-month postoperative measurements was 0.06 $\mathrm{mm}$ (range, -0.34 to $0.46 \mathrm{~mm} ; \mathrm{P}=0.755$ ) and $0.57^{\circ}$ (range, $-0.76^{\circ}$ to $1.89^{\circ} ; \mathrm{P}=0.366$ ), respectively. There was no migration of the grafted tendon or instability of the bone block over the first 3 postoperative months (Table 4).

\section{DISCUSSION}

We found that the clinical outcome did not significantly differ between the interference screw fixation group and the suspensory fixation group after ACL reconstruction using an autogenous BPTB graft. The functional outcome (an KT-2000 arthrometer measurement, the Pivot shift, 
the Lysholm score, the IKDC subjective and objective scores, and the Tegner activity score) and the radiologic outcome (CBD and $\mathrm{RAB}$ ) did not significantly differ between the two treatment groups. Neither migration of the grafted tendon nor instability of the bone block was observed in the patients postoperatively.

Previously, Tecklenburg et al. [7] reported satisfactory results after a 2- to 5-year follow-up in patients who underwent femoral cortical suspensory fixation with an Endobutton for autogenous patellar tendon-bone grafting. Taketomi et al. [17] similarly reported that most bone plugs integrated into the femoral tunnel and that the incidence of graft migration was low in patients who underwent femoral suspensory fixation using ВРТВ grafts in ACL reconstruction. In cases of migration, they found that the distance was short after a 1-year follow-up [17]. However, these were retrospective studies, and no prospective studies have investigated the effect of adjustable loop devices on minimizing the length mismatch between the patellar tendon and the tunnel.

Recent biomechanical studies show that the normal biomechanics of the graft tendon is reproducible after an anatomical placement of the femoral tunnel during ACL reconstruction [1,2,18-20]. The anatomical placement requires a horizontalization of the femoral tunnel, causing the femoral and tibial tunnels to shorten. This encourages a length mismatch between the patellar tendon graft and the tunnel, which is exacerbated with the conventional interference screw fixation technique. In comparison, suspensory fixation of the graft may resolve the graft-tunnel length problem by providing a more sufficient graft tension achieved through an adjustable loop that pulls the graft ends towards the bone tunnel and eliminates free space within [21].

The stability of BPTB grafts after ACL reconstruction using suspensory fixation have not yet been investigated; few studies have investigated suspensory fixation as a femoral fixation tool in ACL reconstruction using BPTB [22]. We hypothesized that adjustable femoral suspensory fixation may help resolve the length mismatch between the grafted tendon and tunnel. We also hypothesized that the outcome of this technique is comparable to those of the conventional interference screw fixation technique.

We assessed graft stability in terms of CBD and RAB. In particular, early migration of the graft-bone block is an important factor because it can lead to loss in tension of the grafted material. Breakage, detachment, or failed penetration of Fiberwire usually causes migration of the graft-bone block [17]. Previous studies have reported that in ACL reconstruction using the transtibial technique a single-strand No. 2 Fiberwire has a failure load of $282 \pm$ $30 \mathrm{~N}$ [23] and an Endobutton CL (Smith \& Nephew Endoscopy) has a failure load of $959.9 \pm 190.4 \mathrm{~N}$ [24]. In this study, we inserted two strands of Fiberwire through a bone plug hole and the four loops of the Fiberwires into an Endobutton, which resulted in a structure that could withstand a maximum force that was up to eight times the force that a single-strand No. 2 Fiberwire can withstand (and much larger than the failure load of an Endobutton CL, the standard suspensory fixation device). Thus, we argue that this apposition of the graft-bone block and the Endobutton provides adequate initial fixation force.

Soft-tissue interposition between the Endobutton and the outer femoral cortex is another factor that can lead to graft migration after femoral suspensory fixation of BPTB grafts in ACL reconstruction. For example, Mae et al. [25] who observed this in $25.2 \%$ of patients who underwent ACL reconstruction using femoral suspensory fixation reported that the incidence of migration was higher in patients with soft-tissue interposition than in those without. However, they also mentioned that the incidence could depend on the operator's level of expertise. In this study, we observed a lower incidence of soft-tissue interposition probably because we made a stab wound on the lateral aspect of the femur to insert the trocar and fixed the Endobutton only after checking its gross position on the femoral cortex.

Limitations to this study warrant review before conclusions can be drawn from our findings. First, the 3-month follow-up period may be insufficient to judge whether the tunnel widening after suspensory fixation occurred because of a bungee effect or a windshield-wiper effect [26]. Taketomi et al. [17] reported that Endobuttons rotated postoperatively in about $56 \%$ of patients who underwent suspensory fixation, suggesting the possibility of graftbone block loosening within femoral tunnels. Suzuki et al. [27] also reported that it takes approximately 2 months for a bone block to integrate into the femoral tunnel after interference screw fixation using BPTB grafts. Although block integration may take longer after suspensory fixation, 3 months is nevertheless regarded to be sufficient to determine whether there has been successful boneblock grafting. Here, we confirmed successful boneblock grafting within the 3-month follow-up in all the patients. Second, our study did not have a randomized 
study design, meaning that patients with similar demographic variables may have been grouped together. Thus, randomization of subjects into groups unrelated to patellar tendon length is necessary to obtain more reliable results. Third, the study population was small. Although we demonstrated using statistical analysis that the minimum sample size needed to compare our primary endpoint—anterior instability—was 13 , this sample size fails to achieve sufficient statistical power when comparing our secondary outcomes, such as clinical scores and radiologic outcomes. Therefore, studies with larger sample sizes are necessary in the future.

In conclusion, we found that the degree of anterior translation of the graft after suspensory fixation of the femoral tunnel in ACL reconstruction was comparable to that after interference screw fixation. Early postoperative loosening of the graft-bone block was not observed upon
CT analysis. Therefore, the findings of this study suggest that suspensory fixation could be an alternative technique to conventional interference screw fixation that overcomes graft-tunnel mismatch in ACL reconstruction using BPTB grafts.

\section{CONFLICT OF INTEREST}

No potential conflict of interest relevant to this article was reported.

\section{ACKNOWLEDGMENTS}

This study was supported by a faculty research grant of Yonsei University College of Medicine for 2010 (6-20100196).

\section{REFERENCES}

1. Yagi M, Wong EK, Kanamori A, Debski RE, Fu FH, Woo SL. Biomechanical analysis of an anatomic anterior cruciate ligament reconstruction. Am J Sports Med 2002;30:660-6.

2. Loh JC, Fukuda Y, Tsuda E, Steadman RJ, Fu FH, Woo SL. Knee stability and graft function following anterior cruciate ligament reconstruction: comparison between 11 o'clock and 10 o'clock femoral tunnel placement. 2002 Richard O'Connor Award paper. Arthroscopy 2003;19:297-304.

3. Scopp JM, Jasper LE, Belkoff SM, Moorman CT 3rd. The effect of oblique femoral tunnel placement on rotational constraint of the knee reconstructed using patellar tendon autografts. Arthroscopy 2004;20:294-9.

4. Heming JF, Rand J, Steiner ME. Anatomical limitations of transtibial drilling in anterior cruciate ligament reconstruction. Am J Sports Med 2007;35:1708-15.

5. Golish SR, Baumfeld JA, Schoderbek RJ, Miller MD. The effect of femoral tunnel starting position on tunnel length in anterior cruciate ligament reconstruction: a cadaveric study. Arthroscopy 2007;23:1187-92.

6. Brand J Jr, Weiler A, Caborn DN, Brown CH Jr, Johnson DL. Graft fixation in cruciate ligament reconstruction. Am J Sports Med 2000;28:761-74.

7. Tecklenburg K, Hoser C, Sailer R, Oberladstätter J, Fink C. ACL reconstruction with bone-patellar tendon-bone graft and proximal fixation with the EndoButton: a 2- to 5-year follow-up. Unfallchirurg 2005;108:721-7.

8. Grawe B, Smerina A, Allen A. Avoiding graft-tunnel length mismatch in anterior cruciate ligament reconstruction: the singlebone plug technique. Arthrosc Tech 2014;3:e417-20.

9. Kim SJ, Kim HJ. High portal: practical philosophy for positioning portals in knee arthroscopy. Arthroscopy 2001;17:333-7.
10. Lee JK, Lee S, Seong SC, Lee MC. Anatomic single-bundle ACL reconstruction is possible with use of the modified transtibial technique: a comparison with the anteromedial transportal technique. J Bone Joint Surg Am 2014;96:664-72.

11. Lee SR, Jang HW, Lee DW, Nam SW, Ha JK, Kim JG. Evaluation of femoral tunnel positioning using 3-dimensional computed tomography and radiographs after single bundle anterior cruciate ligament reconstruction with modified transtibial technique. Clin Orthop Surg 2013;5:188-94.

12. Beighton P, Solomon L, Soskolne CL. Articular mobility in an African population. Ann Rheum Dis 1973;32:413-8.

13. Kim SJ, Kumar P, Oh KS. Anterior cruciate ligament reconstruction: autogenous quadriceps tendon-bone compared with bonepatellar tendon-bone grafts at 2-year follow-up. Arthroscopy 2009;25:137-44.

14. Maletis GB, Cameron SL, Tengan JJ, Burchette RJ. A prospective randomized study of anterior cruciate ligament reconstruction: a comparison of patellar tendon and quadruple-strand semitendinosus/gracilis tendons fixed with bioabsorbable interference screws. Am J Sports Med 2007;35:384-94.

15. Mohtadi NG, Chan DS, Dainty KN, Whelan DB. Patellar tendon versus hamstring tendon autograft for anterior cruciate ligament rupture in adults. Cochrane Database Syst Rev 2011;(9):CD005960.

16. Julious SA. Sample sizes for clinical trials with normal data. Stat Med 2004;23:1921-86.

17. Taketomi S, Inui H, Nakamura K, et al. Secure fixation of femoral bone plug with a suspensory button in anatomical anterior cruciate ligament reconstruction with bone-patellar tendon-bone graft. Joints 2016;3:102-8.

18. Ho JY, Gardiner A, Shah V, Steiner ME. Equal kinematics between 
central anatomic single-bundle and double-bundle anterior cruciate ligament reconstructions. Arthroscopy 2009;25:464-72.

19. Dhawan A, Gallo RA, Lynch SA. Anatomic tunnel placement in anterior cruciate ligament reconstruction. J Am Acad Orthop Surg 2016;24:443-54.

20. Westermann RW, Wolf BR, Elkins J. Optimizing graft placement in anterior cruciate ligament reconstruction: a finite element analysis. J Knee Surg 2017;30:97-106.

21. Yoshiya S. Editorial commentary: controversy on fixation properties of the adjustable-loop cortical suspension fixation device used for anterior cruciate ligament reconstruction. Arthroscopy 2016;32:262.

22. Smith PA, Stannard JP, Pfeiffer FM, Kuroki K, Bozynski CC, Cook $\mathrm{JL}$. Suspensory versus interference screw fixation for arthroscopic anterior cruciate ligament reconstruction in a translational large-animal model. Arthroscopy 2016;32:1086-97.

23. Najibi S, Banglmeier R, Matta J, Tannast M. Material properties of common suture materials in orthopaedic surgery. lowa Orthop
J 2010;30:84-8.

24. Miller CD, Gerdeman AC, Bennett CG, Hart JM, Miller MD. A biomechanical comparison of the endobutton $\mathrm{Cl}$ using transtibial drilling and endobutton direct using anteromedial arthroscopic drilling. Arthroscopy 2010;26:1311-7.

25. Mae T, Kuroda S, Matsumoto N, et al. Migration of EndoButton after anatomic double-bundle anterior cruciate ligament reconstruction. Arthroscopy 2011;27:1528-35.

26. O'Brien SJ, Warren RF, Pavlov H, Panariello R, Wickiewicz TL. Reconstruction of the chronically insufficient anterior cruciate ligament with the central third of the patellar ligament. J Bone Joint Surg Am 1991;73:278-86.

27. Suzuki T, Shino K, Nakagawa S, et al. Early integration of a bone plug in the femoral tunnel in rectangular tunnel ACL reconstruction with a bone-patellar tendon-bone graft: a prospective computed tomography analysis. Knee Surg Sports Traumatol Arthrosc 2011;19 Suppl 1:S29-35. 